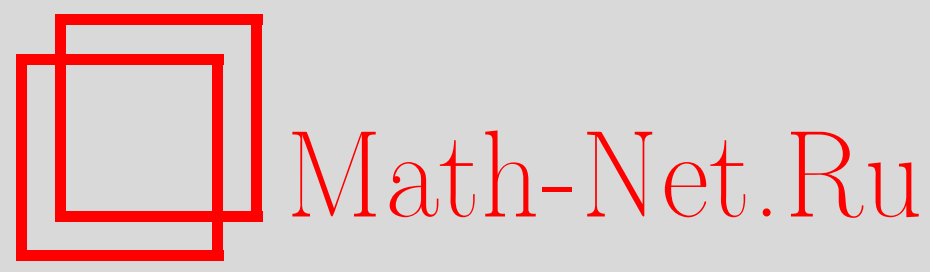

P. М. Тригуб, О приближении гладких функций и констант многочленами с целыми и натуральными коэффициентами, Матем. заметки, 2001, том 70, выпуск 1, 123-136

DOI: https://doi.org/10.4213/mzm725

Использование Общероссийского математического портала Math-Net.Ru подразумевает, что вы прочитали и согласны с пользовательским соглашением http://www.mathnet.ru/rus/agreement

Параметры загрузки:

IP : 54.164 .48 .24

26 апреля 2023 г., 15:40:25

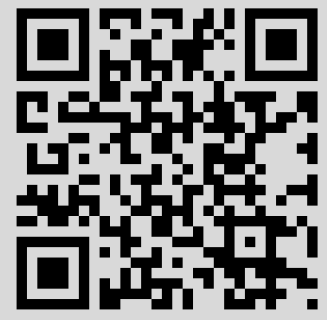




\section{О ПРИБЛИЖЕНИИ ГЛАДКИХ ФУНКЦИЙ И КОНСТАНТ МНОГОЧЛЕНАМИ С ЦЕЛЫМИ И \\ НАТУРАЛЬНЫМИ КОЭФФИЦИЕНТАМИ}

\section{P.M. Тригуб}

Усиливаются прямые теоремы о приближении гладких функций многочленами с цельми коэффициентами. Получены первые теоремы о приближении многочленами с натуральными коэффициентами.

Библиографой: 11 названий.

1. Введение. Формулировка результатов. А. О. Гельфонд [1] доказал аналоги классических теорем Д. Джексона и С. Н. Бернштейна для приближения многочленами с целыми коэффициентами на отрезке $[0,1]$. Общие прямые теоремы о целочисленных приближениях для любого отрезка длины меньше четырех получены автором [2] (см. также обзорные статьи [3] и [4]).

В настоящей статье доказывается следующий асимптотически точный результат.

Теорема 1. Пусть при некотором $r \in \mathbb{N}$ производная $f^{(r-1)}$ абсолютно непрерьвна и $\left|f^{(r)}(x)\right| \leqslant 1$ почти всюду на $[0,1], a f^{(\nu)}(0) / \nu !$ u $f^{(\nu)}(1) / \nu ! \in \mathbb{Z} n p u$ $0 \leqslant \nu \leqslant r-1$ (эти арифметические условия и необходимы). Тогда для любого $n \geqslant 4 r+2$ можно построить многочлен $q_{n}$ степени не выше $n$ с чельми коэффиииентами такой, что $\forall x \in[0,1]$

$$
\left|f(x)-q_{n}(x)\right| \leqslant K_{r}\left(\frac{\sqrt{x(1-x)}}{n}\right)^{r}+\gamma(r) \frac{(\sqrt{x(1-x)})^{r-1}}{n^{r+1}},
$$

где постоянная $\gamma(r)$ зависит лишь от $r$, а константу

$$
K_{r}:=\frac{4}{\pi} \sum_{k=0}^{\infty} \frac{(-1)^{k(r+1)}}{(2 k+1)^{r+1}}
$$

нельзя заменить меньшей.

Без ограничений на коэффициенты эта теорема получена в [5] (в случае $r=1-$ С.М. Никольским, В.Н. Темляковьм).

Приближение функций многочленами с положительными коэффициентами - новая тема, возникшая в связи с одной задачей о спектре положительного оператора [6] (см. также [7]). 
Tеорема 2. Пусть $r \in \mathbb{Z}_{+}:=\mathbb{Z} \cap[0, \infty)$. Ecлu $f \in C^{r}[-2,0], a f^{(\nu)}(0) / \nu ! \in \mathbb{Z}_{+}$ $(0 \leqslant \nu \leqslant r), f^{(\nu)}(-1) / \nu ! \in \mathbb{Z} \quad(0 \leqslant \nu \leqslant r) u$

$$
2^{\nu-r-1}\left(\frac{f^{(\nu)}(-2)}{\nu !}-\sum_{k=\nu}^{r} \frac{f^{(k)}(0)}{k !}\left(\begin{array}{l}
k \\
\nu
\end{array}\right)(-2)^{k-\nu}\right) \in \mathbb{Z} \quad(0 \leqslant \nu \leqslant r)
$$

(все эти арифметические условия и необходимы), то для любого $n \geqslant 3 r+3$ можно построить многочлен $q_{n}^{+}$с коэффичиентами из $\mathbb{Z}_{+}$, удовлетворяющий $\forall x \in[-2,0]$ неравенствам

$$
\left|f^{(\nu)}(x)-\left(q_{n}^{+}\right)^{(\nu)}(x)\right| \leqslant \gamma(r)\left(\frac{\sqrt{|x|(x+2)}}{n}\right)^{r-\nu}\left(\omega\left(f^{(r)} ; \frac{\sqrt{|x|(x+2)}}{n}\right)+\frac{\sqrt{|x|(x+2)}}{n}\right)
$$

$(0 \leqslant \nu \leqslant r)$,

где $\omega\left(f^{(r)} ; h\right)$ - модуль непрерывности $f^{(r)}$ шага $h \geqslant 0$.

Без ограничений на коэффициенты эта теорема получена С. А. Теляковским (см. [8], $\nu=0)$ и И. Е. Гопенгаузом [9] (независимо). Здесь добавлено, что при некоторых условиях на отрезок и функцию коэффициенты приближающих многочленов можно считать целыми и положительными.

Пусть теперь $\lambda \in(-1,1)$ и $\lambda \neq 0$, а отрезок $[a, b] \subset(-1,0)$. Полагаем

$$
E_{n}(\lambda ; a, b ; W):=\min _{\substack{c_{k} \in W \\ 0 \leqslant k \leqslant n}} \max _{[a, b]}\left|\lambda-\sum_{k=0}^{n} c_{k} x^{k}\right|
$$

Вопрос о наилучшем приближении константы многочленами с целыми коэффициентами $(W=\mathbb{Z})$ ставил Бернштейн (см. [2]-[4]). В следующей теореме выделены случаи, когда порядок убьвания последовательности $E_{n}\left(\lambda ; a, b ; \mathbb{Z}_{+}\right)$при $n \rightarrow \infty$ удалось найти.

Tеорема 3. I. Ecлu $|b|(1-a)^{2}<|a|(1+a)^{2}$ uлu $a+b=-1 / q, q \in \mathbb{N}, u \sqrt{|a+b|} \times$ $(\sqrt{|a|}+\sqrt{|b|})<2(\sqrt{|a|}-\sqrt{|b|})$, mo

$$
E_{n}\left(\lambda ; a, b ; \mathbb{Z}_{+}\right) \asymp\left(\frac{\sqrt{|a|}-\sqrt{|b|}}{\sqrt{|a|}+\sqrt{|b|}}\right)^{n}
$$

(двустороннее неравенство с положительными константами, не зависящими om $n)$.

II. Ecлu $|b|(1-a)^{2}>|a|(1+a)^{2}$ u $a=-1 / q$, mo $\forall \lambda \neq \pm p / q^{s} \quad(p, q, s \in \mathbb{N}) n p u$ бесконечно многих $n$

$$
E_{n}\left(\lambda ; a, b ; \mathbb{Z}_{+}\right) \asymp|a|^{n}
$$

(оченка сверху справедлива $\forall n$ ).

III. Если $0.1<|b|<0.5 \leqslant|a| \leqslant 1+b u \lambda \neq p / 2^{s}$, то при бесконечно многих $n$

$$
E_{n}\left(\lambda ; a, b ; \mathbb{Z}_{+}\right) \asymp 2^{-n}
$$

(оченка сверху справедлива $\forall n$ ).

IV. Ecлu $\lambda=p / q^{s}, a+b=-2 / q, b=-r /(r q+1) \quad(p, q, s, r \in \mathbb{N})$, mo $\forall n$

$$
E_{n}\left(\lambda ; a, b ; \mathbb{Z}_{+}\right) \asymp\left(\frac{b-a}{|a+b|}\right)^{n} \text {. }
$$


2. Вспомогательные утверждения. Для доказательства приведенных теорем воспользуемся некоторьми результатами о приближении многочленами $p_{n}$ степени не выше $n$, начиная с известной теоремы А. $\Phi$. Тимана и неравенства Дзядыка-ЛебедяБрудного для производной многочлена (см. [10], а также [11]). Понадобятся также экстремальные и арифметические свойства полиномов Чебьшева (см., например, ниже лемму 6).

Через $\gamma(a, b, c)$ обозначаем константы, зависящие лиш от $a, b$ и $c$. Для любого $n \in \mathbb{N}$ и $x \in[-1,1]$ полагаем

$$
\delta_{n}(x):=\frac{\sqrt{1-x^{2}}}{n}+\frac{1}{n^{2}}, \quad \delta_{n, \circ}(x):=\frac{\sqrt{1-x^{2}}}{n} .
$$

ЛЕмма 1. (См. [2, лемма 1 и замечание 2].) $\quad$ Если $f \in C^{r}[-1,1] u \forall n \in \mathbb{N} u$ $\forall x \in[-1,1]$

$$
\left|f(x)-p_{n}(x)\right| \leqslant \delta_{n}^{r}(x) \omega\left(f^{(r)} ; \delta_{n}(x)\right),
$$

то при некоторой константе $\gamma=\gamma(r) \quad \forall \nu \in[0, r]$

$$
\left|f^{(\nu)}(x)-p_{n}^{(\nu)}(x)\right| \leqslant \gamma \delta_{n}^{r-\nu}(x) \omega\left(f^{(r)} ; \delta_{n}(x)\right), \quad\left|p_{n}^{(r+1)}(x)\right| \leqslant \gamma \frac{\omega\left(f^{(r)} ; \delta_{n}(x)\right)}{\delta_{n}(x)} .
$$

Лемма 2. Для любих $m, k u n \in \mathbb{N}$ можнно построить многочлен $p_{n}$ степенu $\leqslant n$ такой, что $\forall x \in[0,1]$

$$
\left|x^{m}-x^{m+k} p_{n}(x)\right| \leqslant \gamma(m, k) \min \left\{\frac{1}{n^{2 m}}, x^{m}\right\} .
$$

Это неравенство с оценкой $\gamma n^{-2 m}$ получено в [2, лемма $\left.2^{\prime \prime}\right]$. Но тогда в силу неравенства А. А. Маркова для производной (см., например, [10]) $m$-я производная многочлена, стоящего под знаком модуля, ограничена по $n$ и в силу формулы Тейлора имеем оценкy $\gamma x^{m}$.

Лемма 3. Пусть $X$ - многочлен с иелыми коэффициентами $u 0 \leqslant X(x)<1$ $\forall x \in[a, b]$. Если многочлен $p_{n}$ делится без остатка на $X^{m}$ при некотором $m \in \mathbb{N}$, то существует многочлен $q_{n}$ с иелыми коэффиииентами такой, что $\forall x \in[a, b]$

$$
\left|p_{n}(x)-X^{m}(x) q_{n}(x)\right| \leqslant \frac{\gamma}{n^{2 m}},
$$

где константа $\gamma$ зависит лишь от $a, b, m u X$.

ДокАЗАТЕльство. Следует из лемм $2^{\prime \prime}$ и 3 статьи [2].

ЛеммА 4. І. Если многочлен $p_{n}$ удовлетворяет неравенству $(1), p_{n}^{(\nu)}(-1)=$ $f^{(\nu)}(-1), p_{n}^{(\nu)}(1)=f^{(\nu)}(1) \quad(0 \leqslant \nu \leqslant r), m o \forall x \in[-1,1] u \forall \nu \in[0, r]$

$$
\left|f^{(\nu)}(x)-p_{n}^{(\nu)}(x)\right| \leqslant \gamma(r) \delta_{n, \circ}^{r-\nu}(x) \omega\left(f^{(r)} ; \delta_{n, \circ}(x)\right) .
$$

II. Если многочлен $p_{n}$ удовлетворяет на $[-1,1]$ неравенству

$$
\left|p_{n}(x)\right| \leqslant \delta_{n}^{r}(x) \omega\left(\delta_{n}(x)\right)
$$

$u p_{n}^{(\nu)}( \pm 1)=0 \quad(0 \leqslant \nu \leqslant r)$, mo npu $x \in[-1,1] u \nu \in[0, r]$

$$
\left|p_{n}^{(\nu)}(x)\right| \leqslant \gamma(r) \delta_{n, \circ}^{r-\nu}(x) \omega\left(\delta_{n, \circ}(x)\right)
$$


ДОКАЗАТЕЛЬСТВО есть, по сути, в [9].

Лемма 5. Пусть $f \in C^{r}[-2,0]$. Eсли $f^{(\nu)}(0) / \nu ! \in \mathbb{Z}_{+}, f^{(\nu)}(-1) / \nu ! \in \mathbb{Z}(0 \leqslant \nu \leqslant r)$ $u$

$$
2^{\nu-r-1}\left(\frac{f^{(\nu)}(-2)}{\nu !}-\sum_{k=\nu}^{r} \frac{f^{(k)}(0)}{k !}\left(\begin{array}{l}
k \\
\nu
\end{array}\right)(-2)^{k-\nu}\right) \in \mathbb{Z}, \quad 0 \leqslant \nu \leqslant r
$$

(это и необходимо), то существует $q_{3 r+3}^{+}$с коэффициентами из $\mathbb{Z}_{+}$(степени $\leqslant 3 r+3)$ такой, что при $\nu \in[0, r]$

$\left(q_{3 r+3}^{+}\right)^{(\nu)}(0)=f^{(\nu)}(0), \quad\left(q_{3 r+3}^{+}\right)^{(\nu)}(-1)=f^{(\nu)}(-1), \quad\left(q_{3 r+3}^{+}\right)^{(\nu)}(-2)=f^{(\nu)}(-2)$.

При этом на $[-2,0]$

$$
\left|\left(q_{3 r+3}^{+}\right)^{(r+1)}(x)\right| \leqslant \gamma(r) \omega\left(f^{(r)} ; 1\right) .
$$

ДокАЗАТЕЛЬСТво. Начнем с необходимости указанных условий. Пусть $q_{m}(x)=$ $\sum_{k=0}^{m} a_{k} x^{k}-$ какой-либо многочлен, совпадающий с функцией и ее производными до порядка $r$ в точках $0,-1$ и $-2\left(a_{k} \in \mathbb{Z}_{+}, 0 \leqslant k \leqslant m\right)$. Необходимость условий в точках 0 и -1 очевидна. А при $m \geqslant r+1$

$$
\begin{aligned}
2^{\nu-r-1}\left(\frac{f^{(\nu)}(-2)}{\nu !}-\sum_{k=\nu}^{r} \frac{f^{(k)}(0)}{k !}\left(\begin{array}{l}
k \\
\nu
\end{array}\right)(-2)^{k-\nu}\right) \\
=2^{\nu-r-1}\left(\frac{q_{m}^{(\nu)}(-2)}{\nu !}-\sum_{k=\nu}^{r} \frac{q_{m}^{(k)}(0)}{k !}\left(\begin{array}{l}
k \\
\nu
\end{array}\right)(-2)^{k-\nu}\right) \\
=2^{\nu-r-1}\left(\sum_{k=\nu}^{m} a_{k}\left(\begin{array}{l}
k \\
\nu
\end{array}\right)(-2)^{k-\nu}-\sum_{k=\nu}^{r} \frac{q_{m}^{(k)}(0)}{k !}\left(\begin{array}{l}
k \\
\nu
\end{array}\right)(-2)^{k-\nu}\right) \\
=\sum_{k=r+1}^{m} a_{k}\left(\begin{array}{l}
k \\
\nu
\end{array}\right)(-2)^{k-r-1} \in \mathbb{Z} .
\end{aligned}
$$

Теперь построим искомый полином $q_{3 r+3}^{+}$сначала только с цельми коэффициентами $\left(q_{3 r+2}\right)$, а затем, повьшая степень на единицу, и с положительными.

Начнем со случая двух узлов интерполяции 0 и -1 с условиями, указанньми в лемме. Запишем эрмитовский многочлен (наименьшей степени $2 r+1$ ) в виде

$$
\sum_{k=0}^{r} \frac{f^{(k)}(0)}{k !} x^{k}+x^{r+1} \sum_{p=0}^{r} b_{p}(x+1)^{p} .
$$

Очевидно, что $\forall p \in[0, r]$

$$
b_{p}=\frac{1}{p !} \cdot \frac{d^{p}}{d x^{p}}\left\{\left(f(x)-\sum_{k=0}^{r} \frac{f^{(k)}(0)}{k !} x^{k}\right) x^{-r-1}\right\}_{x=-1} \in \mathbb{Z}
$$


так что коэффициенты целые, но могут не быть положительными. Увеличим его степень следующим образом, сохраняя интерполящионные свойства:

$$
\sum_{k=0}^{r} \frac{f^{(k)}(0)}{k !} x^{k}+x^{r+1} \sum_{p=0}^{r} b_{p}(x+1)^{p}\left(1+\frac{1}{2}\left(\operatorname{sign} b_{p}-1\right)(x+1)^{r+1-p}\right) .
$$

Все коэффициенты остаются целыми. Но теперь они все положительные, так как таковыми являются коэффициенты многочлена

$$
(x+1)^{r+1}-(x+1)^{p}=(x+1)^{p}\left((x+1)^{r+1-p}-1\right), \quad 0 \leqslant p \leqslant r .
$$

Оценим теперь $(r+1)$-ю производную построенного многочлена. При $r=0$ модуль производной равен $\left|b_{0}\right|$, а $\left|b_{0}\right|=|f(-1)-f(0)|$. Пусть теперь $r \geqslant 1$. Учтем, что при $p \in[0, r-1]$

$$
\frac{d^{p}}{d x^{p}}\left(f(x)-\sum_{k=0}^{r} \frac{f^{(k)}(0)}{k !} x^{k}\right)=\frac{1}{(r-p-1) !} \int_{0}^{x}\left(f^{(r)}(t)-f^{(r)}(0)\right)(x-t)^{r-p-1} d t
$$

и при $x=-1$ модуль левой части не более

$$
\frac{1}{(r-p-1) !} \int_{-1}^{0}\left|f^{(r)}(t)-f^{(r)}(0)\right|(t+1)^{r-p-1} d t \leqslant \frac{1}{(r-p) !} \omega\left(f^{(r)} ; 1\right) .
$$

При $p=r$ такая оценка модуля левой части очевидна. Поэтому (см. (2)) $\forall p \in[0, r]$ $\left|b_{p}\right| \leqslant \gamma_{1}(r) \omega\left(f^{(r)} ; 1\right)$. Но тогда в силу $(3)$ модуль $(r+1)$-й производной не более $\gamma_{2}(r) \times$ $\omega\left(f^{(r)} ; 1\right)$.

Теперь возьмем два узла 0 и -2 с условиями, указанными в лемме. Введем вспомогательную функцию

$$
\tilde{f}(x):=\frac{1}{2^{r+1}}\left(f(2 x)-\sum_{k=0}^{r} \frac{f^{(k)}(0)}{k !}(2 x)^{k}\right)
$$

и построим для нее многочлен $\tilde{q}$ по двум узлам 0 и -1 (см. вьше). При $\nu \in[0, r]$ $\tilde{f}^{(\nu)}(0)=0$ и

$$
\frac{\tilde{f}^{(\nu)}(-1)}{\nu !}=\frac{1}{2^{r+1}}\left(2^{\nu} \cdot \frac{f^{(\nu)}(-2)}{\nu !}-\sum_{k=\nu}^{r} \frac{f^{(k)}(0)}{k !}\left(\begin{array}{l}
k \\
\nu
\end{array}\right) 2^{\nu}(-2)^{k-\nu}\right) \in \mathbb{Z} .
$$

Коэффициенты $\tilde{q}$ из $\mathbb{Z}_{+}$, а степень его $2 r+2$. Но тогда искомьй многочлен в случае узлов 0 и -2

$$
q(x)=2^{r+1} \tilde{q}\left(\frac{x}{2}\right)+\sum_{k=0}^{r} \frac{f^{(k)}(0)}{k !} x^{k} .
$$

Действительно, $q^{(\nu)}(0)=f^{(\nu)}(0)(0 \leqslant \nu \leqslant r)$ и при $\nu \in[0, r]$

$$
\frac{q^{(\nu)}(-2)}{\nu !}=2^{r+1-\nu} \cdot \frac{\tilde{f}^{(\nu)}(-1)}{\nu !}+\sum_{k=\nu}^{r} \frac{f^{(k)}(0)}{k !}\left(\begin{array}{l}
k \\
\nu
\end{array}\right)(-2)^{k-\nu}=\frac{f^{(\nu)}(-2)}{\nu !}
$$


а все его коэффициенты, как и у $\tilde{q}$, из $\mathbb{Z}_{+}$. Если этот многочлен, построенньй по узлам 0 и -2 , обозначить через $q_{2 r+2}^{+}$, то искомый многочлен $q_{3 r+3}^{+}$для трех узлов равен

$$
q_{2 r+2}^{+}(x)+(x(x+2))^{r+1} \sum_{p=0}^{r} b_{p}(x+1)^{p}\left(1+\frac{1}{2}\left(\operatorname{sign} b_{p}-1\right)(x+1)^{r+1-p}\right),
$$

где при $p \in[0, r]$

$$
b_{p}=\frac{1}{p !} \cdot \frac{d^{p}}{d x^{p}}\left\{\left(f(x)-q_{2 r+2}^{+}(x)\right)(x(x+2))^{-r-1}\right\}_{x=-1} \in \mathbb{Z} .
$$

Но так как $\left(q_{2 r+2}^{+}\right)^{(\nu)}(0)=f^{(\nu)}(0)$ при $\nu \in[0, r]$ и $\left|\left(q_{2 r+2}^{+}\right)^{(r+1)}(x)\right| \leqslant \gamma_{3} \omega\left(f^{(r)} ; 1\right)$, то, как и при доказательстве (4), получаем $\forall p \in[0, r]$

$$
\begin{aligned}
\left|f^{(p)}(-1)-\left(q_{2 r+2}^{+}\right)^{(p)}(-1)\right| & \leqslant \frac{\omega\left(\left(f-q_{2 r+2}^{+}\right)^{(r)} ; 1\right)}{(r-p) !} \leqslant \frac{\omega\left(f^{(r)} ; 1\right)+\omega\left(\left(q_{2 r+2}^{+}\right)^{(r)} ; 1\right)}{(r-p) !} \\
& \leqslant \frac{\omega\left(f^{(r)} ; 1\right)+\max \left|\left(q_{2 r+2}^{+}\right)^{(r+1)}(x)\right|}{(r-p) !} \leqslant \gamma_{4} \omega\left(f^{(r)} ; 1\right) .
\end{aligned}
$$

Следовательно, такую же оценку имеем и для $\left(q_{3 r+3}^{+}\right)^{(r+1)}$.

ЛЕмма 6. Для любого $n \in \mathbb{N}$ существует многочлен $q_{n}^{+}$с натуральными коэффиииентами такой, что $\forall x \in[-2,0]$

$$
\left|2 x+x^{2} q_{n}^{+}(x)\right| \leqslant C \min \left\{\frac{1}{n^{2}},|x(x+1)(x+2)|\right\},
$$

где $C$ - константа абсолютная.

ДокАЗАТЕЛЬСТво. При $n=1$ можно положить $q_{1}^{+}(x)=x+3$, так как $2 x+q_{1}^{+}(x)=$ $x(x+1)(x+2)$. Очевидно, что можно ограничиться случаем, когда $n$ принимает значения членов бесконечной геометрической прогрессии.

Пусть полином Чебышева (см. [10]) для отрезка $[-1,1]$ равен

$$
T_{n}(x):=\cos (n \arccos x)=\frac{1}{2}\left[\left(x+\sqrt{x^{2}-1}\right)^{n}+\left(x-\sqrt{x^{2}-1}\right)^{n}\right] .
$$

Применяя формулу бинома, легко видеть, что $T_{n}^{(\nu)}(1) / \nu ! \in \mathbb{N}(0 \leqslant \nu \leqslant n)$. Убедимся в том, и это главное, что при $k \in[1, n]$ и $n=2^{s} \quad(s=0,1, \ldots)$

$$
\frac{2}{k !} \cdot \frac{T_{n}^{(k)}(1)}{T_{n}^{\prime}(1)}=\frac{2}{n^{2}} \cdot \frac{1}{k !} T_{n}^{(k)}(1) \in \mathbb{N} .
$$

При $n=1$ и $n=2$ проверяем непосредственно:

$$
T_{1}(x)=x, \quad T_{2}(x)=2 x^{2}-1=2(x-1)^{2}+4(x-1)+1,
$$


а далее проводим индукцию по $s$ : от $n$ к $2 n$. Из формулы удвоения угла для косинуса следует, что $T_{2 n}(x)=2 T_{n}^{2}(x)-1$, откуда

$$
\begin{aligned}
\frac{2}{4 n^{2}} \cdot \frac{1}{k !} T_{2 n}^{(k)}(1) & =\frac{1}{n^{2}} \sum_{\nu=0}^{k} \frac{T_{n}^{(\nu)}(1)}{\nu !} \cdot \frac{T_{n}^{(k-\nu)}(1)}{(k-\nu) !} \\
& =\sum_{\nu=0}^{[(k-1) / 2]} \frac{T_{n}^{(\nu)}(1)}{\nu !}\left(\frac{2}{n^{2}} \cdot \frac{T_{n}^{(k-\nu)}(1)}{(k-\nu) !}\right)+\frac{1+(-1)^{k}}{2 n^{2}}\left(\frac{T_{n}^{[k / 2]}(1)}{([k / 2]) !}\right)^{2} .
\end{aligned}
$$

При $k=1 T_{n}^{\prime}(1)=n^{2}$. А при $k \geqslant 2$ в силу предположения индукции сумма равна натуральному числу. И последнее слагаемое (при четном $k$ )

$$
\left(\frac{2}{n^{2}} \cdot \frac{1}{(k / 2) !} \cdot T_{n}^{(k / 2)}(1)\right)^{2} \cdot \frac{n^{2}}{4} \in \mathbb{N} \text {. }
$$

Соотношение (6) установлено.

А теперь при $n=2^{s}$ полагаем

$$
2 x+x^{2} q_{n}^{+}(x)=\frac{2}{n^{2}}\left(T_{n}(x+1)-T_{n}(1)\right)=\sum_{k=1}^{n} \frac{2}{n^{2}} \cdot \frac{T_{n}^{(k)}(1)}{k !} x^{k} .
$$

При $s \geqslant 2$ этот многочлен равен нулю для $x=0,-1$ и -2 . Очевидно, что при $x \in[-2,0]$ модуль его не более $4 / n^{2}$, а его производная по модулю не более двух. Поэтому еще $\forall x \in[-2,0]$

$$
\left|2 x+x^{2} q_{n}^{+}(x)\right| \leqslant 2 \min \{|x|,|x+1|,|x+2|\} \leqslant \frac{8}{3}|x(x+1)(x+2)| .
$$

Таким образом, можно считать в лемме $6 C=16$.

Заметим, что в утверждении леммы 6 заменить $2 x$ на $x$ нельзя, как видно после подстановки $x=-2$.

ЛЕмма 7. І. Пусть $q^{+}$- многочлен степени $s$ скоэффициентами из $\mathbb{Z}_{+} u$ $c \leqslant q^{+}(x) \leqslant d<0 \quad \forall x \in[a, b] \subset(-1,0)$. Тогдa $\forall n$

$$
E_{n}\left(\lambda ; a, b ; \mathbb{Z}_{+}\right) \leqslant\left(\left[\frac{n}{s}\right]+1\right) \rho^{s[n / s]}, \quad \rho=\max \left\{|c|, \frac{\sqrt{|c|}-\sqrt{|d|}}{\sqrt{|c|}+\sqrt{|d|}}\right\}^{1 / s} .
$$

$B$ случае $|c|(\sqrt{|c|}+\sqrt{|d|}) \neq \sqrt{|c|}-\sqrt{|d|}$ множситель $\left[\frac{n}{s}\right]+1$ можно заменить константой.

II. $\forall \lambda \in(0,1 / 2]$ при любом $n \in \mathbb{N}$

$$
E_{n}(\lambda ; a, b ; \mathbb{Z}) \geqslant \lambda\left(\frac{\sqrt{|a|}-\sqrt{|b|}}{\sqrt{|a|}+\sqrt{|b|}}\right)^{n}
$$


ДоКАЗАТЕЛЬСтво. І. Пусть полином Чебьшева для $[c, d]$ равен

$$
T_{m}(x, c, d)=\cos \left(m \arccos \frac{2 x-c-d}{d-c}\right)=\gamma(m)+x \gamma(m) p_{m-1}^{+}(x) .
$$

Так как все нули $T_{m}$ лежат на $(c, d) \subset(-\infty, 0]$, а старший коэффишиент положительный, то и все его коэффициенты положительные. В частности, $\gamma(m)=T_{m}(0, c, d)>0$. Тогда при $x \in[c, d]$

$$
\left|1+x p_{m-1}^{+}(x)\right| \leqslant \frac{\left|T_{m}(x, c, d)\right|}{\gamma(m)} \leqslant \frac{1}{\gamma(m)} .
$$

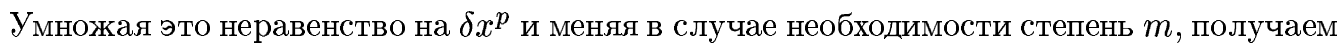
порядок приближения этого одночлена более высокими степенями $x$ с коэффициентами одного знака. Считаем далее $\lambda \in(0,1)$. Случай $\lambda \in(-1,0)$ сводится к предыдущему, так как тогда $\lambda=(\lambda+1)-1$, где $\lambda+1 \in(0,1)$. Любое число $\delta>0$ можно представить в виде $\delta=k-\varepsilon$, где $k \in \mathbb{N}$ и $\varepsilon \in(0,1]$. Полагаем $k=[\delta]$. Заменяя еще $x$ на $q^{+}(x)$, имеем при некоторых числах $a_{1, k}>0$

$$
\begin{aligned}
\mid \lambda- & {[\lambda]+(\lambda-[\lambda]) q^{+}(x) p_{[n / s]-1}^{+}\left(q^{+}(x)\right) \mid } \\
& =\left|\lambda-[\lambda]-\sum_{k=1}^{[n / s]} a_{1, k}\left(q^{+}(x)\right)^{k}\right| \leqslant \frac{1}{\gamma([n / s])}
\end{aligned}
$$

и далее при некоторых $a_{2, k}>0$

$$
\begin{aligned}
\mid \lambda- & {[\lambda]-\left[a_{1,1}\right] q^{+}(x)-\sum_{k=2}^{[n / s]} a_{2, k}\left(q^{+}(x)\right)^{k} \mid } \\
= & \mid\left[\lambda-[\lambda]-\sum_{k=1}^{[n / s]} a_{1, k}\left(q^{+}(x)\right)^{k}\right] \\
& +\left[\left(a_{1,1}-\left[a_{1,1}\right]\right) q^{+}(x)+\left(a_{1,1}-\left[a_{1,1}\right]\right)\left(q^{+}(x)\right)^{2} p_{[n / s]-2}^{+}\left(q^{+}(x)\right)\right] \mid \\
\leqslant & \frac{1}{\gamma([n / s])}+\frac{\left|q^{+}(x)\right|}{\gamma([n / s]-1)} .
\end{aligned}
$$

Продолжая аналогично, получаем

$$
\left|\lambda-[\lambda]-\sum_{k=1}^{n}\left[a_{k, k}\right]\left(q^{+}(x)\right)^{k}\right| \leqslant \sum_{\nu=0}^{[n / s]} \frac{\left|q^{+}(x)\right|^{\nu}}{\gamma([n / s]-\nu)} .
$$

Остается учесть, что на $[a, b]\left|q^{+}(x)\right| \leqslant|c|$, а в силу (5)

$$
\begin{aligned}
\gamma(m) & =T_{m}(0, c, d)=\cos \left(m \arccos \frac{c+d}{c-d}\right) \\
& \geqslant \frac{1}{2}\left(\frac{c+d}{c-d}+\sqrt{\left(\frac{c+d}{c-d}\right)^{2}-1}\right)^{m}=\frac{1}{2}\left(\frac{\sqrt{|c|}+\sqrt{|d|}}{\sqrt{|c|}-\sqrt{|d|}}\right)^{m}
\end{aligned}
$$


и при $\rho$ и $|c| \in(0,1]$

$$
\sum_{\nu=0}^{p}|c|^{\nu} \rho^{p-\nu} \leqslant \max \{|c|, \rho\}^{p} \cdot \min \left\{p+1, \frac{1}{|\rho-| c||}\right\} .
$$

II. Из известного экстремального свойства полиномов Чебышева (см., например, [10, c. 79]) следует с учетом (5)

$$
\lambda \leqslant\left|\lambda-q_{n}(0)\right| \leqslant \max _{[a, b]}\left|\lambda-q_{n}(x)\right| \cdot\left|T_{n}(0, a, b)\right| \leqslant E_{n}(\lambda ; a, b ; \mathbb{Z})\left(\frac{\sqrt{|b|}+\sqrt{|a|}}{\sqrt{|b|}-\sqrt{|a|}}\right)^{n} .
$$

Здесь $q_{n}$ - многочлен наилучшего приближения числа $\lambda$ с целыми коэффициентами.

Лемма 8. I. Если $p / q \in[a, b], a \lambda \neq p_{1} / q^{s} \quad\left(p, q, p_{1}, s \in \mathbb{N}\right)$, то для бесконечной последовательности п

$$
E_{n}\left(\lambda ; a, b: \mathbb{Z}_{+}\right) \geqslant \frac{1}{q^{n+2}} .
$$

II. $E \operatorname{cл} u \lambda=p / q^{s} \quad(p, q, s \in \mathbb{N}) u-2 / q<a<b<0, m o$

$$
E_{n}\left( \pm \lambda ; a, b ; \mathbb{Z}_{+}\right) \leqslant \gamma(s) \max \{|1+a q|,|1+b q|\}^{n} .
$$

ДОКАЗАТЕЛЬСТво. I.

$$
E_{n}\left(\lambda ; a, b ; \mathbb{Z}_{+}\right) \geqslant\left|\lambda-q_{n}\left(\frac{p}{q}\right)\right| \geqslant \inf _{p}\left|\lambda-\frac{p}{q^{n}}\right| \geqslant \frac{1}{q^{n+2}}
$$

для следующей бесконечной последовательности $n$ : после $n$-го знака в $q$-ичном разложении числа $\lambda$ не следует подряд два раза 0 или $q-1$.

II. Положим

$$
q_{n}^{+}(x)=\frac{1}{q}(1+q x)^{n}-\frac{1}{q}=n x+q x^{2} \hat{q}_{n-2}^{+}(x) .
$$

Тогда при $\lambda=p / q \leqslant 1(s=1)$

$$
\left|\frac{p}{q}+p q_{n}^{+}(x)\right| \leqslant \max _{[a, b]}|1+q x|^{n} \leqslant \max \{|1+a q|,|1+b q|\}^{n}
$$

и

$$
\left|\frac{p}{q}-1-(q-p) q_{n}^{+}(x)\right| \leqslant \max _{[a, b]}|1+q x|^{n} \leqslant \max \{|1+a q|,|1+b q|\}^{n} .
$$

Умножая эти неравенства на $1 / q$ и используя их, получаем нужную оценку приближения числа $p / q^{2}(s=2)$ многочленами с коэффициентами из $\mathbb{Z}_{+}$или $-\mathbb{Z}_{+}$. Далее следует индукция по $s$. 
ДОКАЗАТЕЛЬСТВО ТЕОРЕМЫ 1 . В силУ теоремы 1 из [5] для любого $n \geqslant r-1$ существует многочлен $p_{n}$ такой, что $\forall x \in[0,1]$

$$
\left|f(x)-p_{n}(x)\right| \leqslant K_{r}\left(\frac{\sqrt{x(1-x)}}{n}\right)^{r}+\gamma_{1}(r) \frac{(\sqrt{x(1-x)})^{r-1}}{n^{r+1}}
$$

и константу $K_{r}$ заменить меньшей, вообще говоря, нельзя.

Эрмитовский интерполяционньй многочлен $h=h_{2 r-1}$, определяемьй условиями $h^{(\nu)}(0)=f^{(\nu)}(0), h^{(\nu)}(1)=f^{(\nu)}(1) \quad(0 \leqslant \nu \leqslant r-1)$, имеет целые коэффициенты. Применяя лемму 5 , получаем

$$
\left|h^{(r)}(x)\right| \leqslant \gamma_{2}(r) \omega\left(f^{(r-1)} ; 1\right) \leqslant \gamma_{2}(r)\left\|f^{(r)}\right\|_{\infty} \leqslant \gamma_{2}(r)
$$

и $\left\|(f-h)^{(r)}\right\|_{\infty} \leqslant 1+\gamma_{2}(r)$. Отсюда следует,что для всех $x \in[0,1]$

$$
|f(x)-h(x)| \leqslant \gamma_{3}(r)(x(1-x))^{r},
$$

а в силу (7) и

$$
\left|p_{n}(x)-h(x)\right| \leqslant \gamma_{4}(r)(x(1-x))^{(r-1) / 2} .
$$

Заменяя $f$ на $f-h$, считаем далее, что $f^{(\nu)}(0)=f^{(\nu)}(1)=0(0 \leqslant \nu \leqslant r-1)$. Тогда при $r \geqslant 2 p_{n}^{(\nu)}(0)=p_{n}^{(\nu)}(1)=0$ при $0 \leqslant \nu<(r-1) / 2$. С другой стороны, из (7) следует, что $\forall x \in[0,1]$

$$
\left|f(x)-p_{n}(x)\right| \leqslant K_{r}\left(\frac{\sqrt{x(1-x)}}{n}+\frac{\gamma_{1}(r)}{r} \frac{1}{n^{2}}\right)^{r} .
$$

Применим лемму 1 (при $\nu=r$ равенство почти всюду):

$$
\left|f^{(\nu)}(x)-p_{n}^{(\nu)}(x)\right| \leqslant \gamma_{5}(r)\left(\frac{\sqrt{x(1-x)}}{n}+\frac{1}{n^{2}}\right)^{r-\nu}, \quad 0 \leqslant \nu \leqslant r .
$$

Отсюда получаем, подставляя $x=0$ и $x=1$,

$$
\left|p_{n}^{(\nu)}(0)\right|+\left|p_{n}^{(\nu)}(1)\right| \leqslant \gamma_{6}(r) n^{2(\nu-r)}, \quad \frac{r-1}{2} \leqslant \nu \leqslant r .
$$

А так как еще $\left|p_{n}^{(r)}(x)\right| \leqslant \gamma_{7}(r) \forall x \in[0,1]$, то в силу неравенства А. А. Маркова и при $r<\nu<2 r \forall x \in[0,1]$

$$
\left|p_{n}^{(\nu)}(x)\right| \leqslant \gamma_{7}(r) n^{2(\nu-r)} 2^{\nu-r} \leqslant 2^{r} \gamma_{7}(r) n^{2(\nu-r)} .
$$

Положим $X(x):=x(1-x)$. На $[0,1] 0 \leqslant X(x) \leqslant 1 / 4$. Разложим $p_{n}$ по степеням многочлена $X$ :

$$
p_{n}(x)=\sum_{(r-1) / 2 \leqslant k \leqslant N_{1}=[n / 2]}\left(a_{k} x+b_{k}(1-x)\right) X^{k}(x) .
$$


Очевидно, что при $\nu \geqslant(r-1) / 2$

$$
p_{n}^{(\nu)}(0)=\frac{d^{\nu}}{d x^{\nu}}\left\{\sum_{(r-1) / 2 \leqslant k<\nu}\left(a_{k} x+b_{k}(1-x)\right) X^{k}(x)\right\}_{x=0}+\nu ! b_{\nu}
$$

(аналогичное равенство имеет место при $x=1$ с заменой $b_{\nu}$ на $(-1)^{\nu} a_{\nu}$ ). Теперь из (8) и (9) индукцией по $\nu$ получаем, что

$$
\left|a_{k}\right|+\left|b_{k}\right| \leqslant \gamma_{8}(r) n^{2(k-r)}, \quad \frac{r-1}{2} \leqslant k<2 r .
$$

Применим лемму 2 при $m=k, k=2 r-m$ с заменой $n$ на $n-4 r-1$ и $x$ на $X(x)$. При $(r-1) / 2 \leqslant k<2 r$ и $x \in[0,1]$

$$
\begin{aligned}
& \left|\left(a_{k} x+b_{k}(1-x)\right) X^{k}(x)-\left(a_{k} x+b_{k}(1-x)\right) X^{2 r}(x) \tilde{p}_{n-4 r-1}(x)\right| \\
& \quad \leqslant \gamma_{8}(r) n^{2(k-r)} \gamma_{9}(r) \min \left\{\frac{1}{(n-4 r-1)^{2 k}}, X^{k}(x)\right\} \\
& \quad \leqslant \gamma_{10}(r) n^{2(k-r)} \min \left\{\frac{1}{n^{2 k}}, X^{k}(x)\right\} .
\end{aligned}
$$

Учитьвая, что для любых $a \geqslant 0, b \geqslant 0$ и $\varepsilon \in(0,1)$

$$
\min \{a, b\} \leqslant a^{1-\varepsilon} b^{\varepsilon}
$$

и выбирая $\varepsilon=(r-1) /(2 k)$ (при $k=0$ и $r=1$ все очевидно), получаем нужную оценку

$$
\gamma_{10}(r) \frac{1}{n^{r+1}} X^{(r-1) / 2}=\gamma_{10} \frac{(\sqrt{x(1-x)})^{r-1}}{n^{r+1}} .
$$

Таким образом, многочлен $p_{n}$ в (7) можно считать делящимся без остатка на $X^{2 r}(x)$. Но тогда в силу леммы 3 существует многочлен $q_{n-4 r}$ с цельми коэффициентами такой, что на $[0,1]$

$$
\left|p_{n}(x)-X^{2 r}(x) q_{n-4 r}(x)\right| \leqslant \gamma_{11}(r) \frac{1}{n^{2 r}} .
$$

Но многочлен степени не вьше $n$, которьй стоит под знаком модуля, равен нулю при $x=0$ и $x=1$ вместе со своими производными до порядка $2 r-1$, а его производная порядка $2 r$ в силу неравенства А. А. Маркова ограничена по $n$. Поэтому из предыдущего неравенства следует, что

$$
\left|p_{n}(x)-X^{2 r}(x) q_{n-2 r}(x)\right| \leqslant \gamma_{12}(r) \min \left\{\frac{1}{n^{2 r}}, X^{2 r}(x)\right\} \leqslant \gamma_{12} \frac{X^{r-1}(x)}{n^{r+1}} .
$$


ДоКАЗАТЕЛЬСТВо ТЕОРЕМЫ 2. В силу леммы 5 существует многочлен $h$ степени $3 r+3$ с натуральньми коэффициентами и условиями: $h^{(\nu)}(-2)=f^{(\nu)}(-2), h^{(\nu)}(-1)=$ $f^{(\nu)}(-1), h^{(\nu)}(0)=f^{(\nu)}(0) \quad(0 \leqslant \nu \leqslant r)$. Заменяя $f$ на $f-h$, считаем далее, что $f^{(\nu)}(0)=f^{(\nu)}(-1)=f^{(\nu)}(-2)=0(0 \leqslant \nu \leqslant r)$. Так как

$$
\omega(\lambda h) \leqslant(\lambda+1) \omega(h)
$$

(см., например, [10, с. 111]), то в силу той же леммы 5 при $\delta \in(0,1]$

$$
\begin{aligned}
\omega\left((f-h)^{(r)} ; \delta\right) & \leqslant \omega\left(f^{(r)} ; \delta\right)+\omega\left(h^{(r)} ; \delta\right) \leqslant \omega\left(f^{(r)} ; \delta\right)+\delta\left\|h^{(r+1)}\right\|_{\infty} \\
& \leqslant \omega\left(f^{(r)} ; \delta\right)+\gamma(r) \delta \omega\left(f^{(r)} ; 1\right) \leqslant(1+2 \gamma(r)) \omega\left(f^{(r)} ; \delta\right) .
\end{aligned}
$$

Так как

$$
\max _{[-2,0]}|x(x+1)(x+2)|=\frac{2}{3 \sqrt{3}}<1,
$$

то в силу теоремы 1 из [2] существует многочлен $q_{n}$ такой, что $\forall x \in[-2,0]$ (определе-

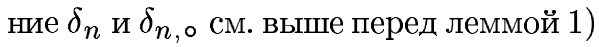

$$
\left|f(x)-q_{n}(x)\right| \leqslant \gamma(r) \delta_{n}^{r}(x+1) \omega\left(f^{(r)} ; \delta_{n}(x+1)\right) .
$$

При этом $q_{n}$ делится без остатка на $X_{1}^{r+1}$, где $X_{1}(x)=x(x+1)(x+2)$. В частности, $q_{n}^{(\nu)}(0)=0=f^{(\nu)}(0)$ и $q_{n}^{(\nu)}(-2)=0=f^{(\nu)}(-2)$ при $0 \leqslant \nu \leqslant r$. А тогда в силу леммы $4(\mathrm{I})$ на самом деле $\forall x \in[-2,0]$ и $\forall \nu \in[0, r]$

$$
\left|f^{(\nu)}(x)-q_{n}^{(\nu)}(x)\right| \leqslant \gamma_{13}(r) \delta_{n, o}^{r-\nu}(x+1) \omega\left(f^{(r)} ; \delta_{n, \circ}(x+1)\right) .
$$

Из (11) еще следует в силу леммы 1 и (10) $\forall x \in[-2,0]$

$$
\left|q_{n}^{(r+1)}(x)\right| \leqslant \gamma_{14}(r) \frac{\omega\left(f^{(r)} ; \delta_{n}(x+1)\right)}{\delta_{n}(x+1)} \leqslant 2 \gamma_{14}(r) \omega\left(f^{(r)} ; \frac{1}{n^{2}}\right) n^{2} .
$$

Отсюда в силу неравенства Маркова (см. [10, с. 240]) $\forall m \in[0, n-r-1]$

$$
\left|q_{n}^{(r+1+m)}(0)\right| \leqslant 2 \gamma_{14} n^{2} \omega\left(f^{(r)} ; \frac{1}{n^{2}}\right) T_{n-r-1}^{(m)}(1)
$$

и, значит, все коэффициенты многочлена

$$
q_{n}(x)+\left(1+\left[2 \gamma_{14} n^{2} \omega\left(f^{(r)} ; \frac{1}{n^{2}}\right)\right]\right) T_{n}(x+1)
$$

целые и неотрицательные, так как еще $T_{n}^{(\nu)}(1) / \nu ! \in \mathbb{N}$ при $0 \leqslant \nu \leqslant n$ и при $m \in[0, n-r-1]$

$$
T_{n-r-1}^{(m)}(1) \leqslant T_{n}^{(m)}(1) \leqslant T_{n}^{(r+1+m)}(1) .
$$


Если теперь $q_{n}^{+}-$многочлен из леммы 6 , то искомьй многочлен имеет вид

$$
q_{n}(x)+\left(1+\left[2 \gamma_{14} n^{2} \omega\left(f^{(r)} ; \frac{1}{n^{2}}\right)\right]\right) T_{n}(x+1)\left(2 x+x^{2} q_{n}^{+}(x)\right)^{r+1} .
$$

Если его обозначить через $\tilde{q}_{N}^{+}$, то $\left(\tilde{q}_{N}^{+}\right)^{(\nu)}(0)=0$ при $0 \leqslant \nu \leqslant r$ и $\left(\tilde{q}_{N}^{+}\right)^{(\nu)}(0) / \nu ! \in \mathbb{N}$ при $r+1 \leqslant \nu \leqslant n(r+2)+2 r+2$. Оценка приближения функции $f$ (см. (11)) изменится на

$$
\left(1+2 \gamma_{14} n^{2} \omega\left(f^{(r)} ; \frac{1}{n^{2}}\right)\right) C^{r+1} \min \left\{\frac{1}{n^{2}} ;|x|(x+2)\right\}^{r+1} .
$$

Если $\sqrt{|x|(x+2)} \geqslant 1 / n$, то в силу монотонности модуля $\omega$ это не больше

$$
\left(C \cdot \frac{\sqrt{|x|(x+2)}}{n}\right)^{r+1}+2 \gamma_{14} C^{r+1}\left(\frac{\sqrt{|x|(x+2)}}{n}\right)^{r} \omega\left(f^{(r)} ; \frac{\sqrt{|x|(x+2)}}{n}\right) .
$$

Если же $\sqrt{|x|(x+2)}<1 / n$, то в силу (10)

$$
n^{2} \omega\left(f^{(r)} ; \frac{1}{n^{2}}\right) \leqslant 2\left(\frac{n}{\sqrt{|x|(x+2)}}\right) \omega\left(f^{(r)} ; \frac{\sqrt{|x|(x+2)}}{n}\right) .
$$

Осталось доказать нужную оценку производной многочлена $\tilde{q}_{N}^{+}-q_{n}$ порядка $\nu \in[1, r]$. Но для этого достаточно применить лемму 4 (II).

Теорема 2 доказана для степеней $n \geqslant 3 r+4$. Интерполящионный многочлен из леммы 5 осушествляет искомоеприближение для наименьшей из возможных степеней $3 r+3$.

ДОКАЗАТЕЛЬСТВо ТЕОРЕМЫ 3. І. Оценка приближения снизу содержится в лемме 7 (II). Положим в лемме 7 (I) $q^{+}(x)=x(c=a, d=b)$. Если $|b|(1-a)^{2}<|a|(1+a)^{2}$, то $|a|(\sqrt{|a|}+\sqrt{|b|}) \leqslant \sqrt{|a|}-\sqrt{|b|}$ и, значит,

$$
E_{n}\left(\lambda ; a, b ; \mathbb{Z}_{+}\right)=O\left(\frac{\sqrt{|a|}-\sqrt{|b|}}{\sqrt{|a|}+\sqrt{|b|}}\right)^{n} .
$$

В случае $a+b=-1 / q$ положим в лемме $7(\mathrm{I}) q^{+}(x)=x(1+q x)$. Тогда $s=2, c=(a+b) / 4$ и $d=a b /(a+b)$. Остается учесть, что при указанном в теореме условии

$$
|c|=\frac{1}{4}|a+b|<\left(\frac{\sqrt{|a|}-\sqrt{|b|}}{\sqrt{|a|}+\sqrt{|b|}}\right)^{2} .
$$

II. Оценка сверху следует из леммы $7(\mathrm{I})$ при $q^{+}(x)=x$, а оценка снизу из леммы $8(\mathrm{I})$. III. Применяем лемму $7(\mathrm{I})$ при $q^{+}(x)=x(1+x)$ и лемму $8(\mathrm{I})$.

IV. Для оценки приближения сверху применяем лемму 8(II), учитывая, что

$$
1+b q=-1-a q=\frac{b-a}{|a+b|} .
$$

Оценка снизу получается из следующего рассуждения:

$$
\begin{aligned}
E_{n}\left(\frac{p}{q^{s}} ; a, b ; \mathbb{Z}\right) & \geqslant \inf _{q_{n}}\left|\frac{p}{q^{s}}-q_{n}(b)\right| \geqslant \inf _{p_{1} \in \mathbb{Z}}\left|\frac{p}{q^{s}}-\frac{p_{1}}{(r q+1)^{n}}\right|=\inf _{p_{1} \in \mathbb{Z}} \frac{\left|p(r q+1)^{n}-p_{1} q^{s}\right|}{q^{s}(r q+1)^{n}} \\
& \geqslant \frac{1}{q^{s}(r q+1)^{n}}=\frac{1}{q^{s}}\left(\frac{b-a}{|a+b|}\right)^{n} .
\end{aligned}
$$

Отметим еще, что, исходя из доказательства теоремы 3 , как и в [2, см. теорему 4], можно оценить разность между наилучшим приближением любой функции многочленами степени $n$ с любыми коэффициентами и только с натуральными. 


\section{СПИСОК ЦИТИРОВАННОЙ ЛИТЕРАТУРЫ}

[1] Гельфонд А. О. О равномерных приближениях многочленами с целыми коэфффициентами // УМН. 1955. Т. 10. № 1. С. 41-65.

[2] Тригуб Р. М. Приближение функций многочленами с целыми коэффициентами // Изв. АН СССР. Сер. матем. 1962. Т. 26. № 2. С. 261-280.

[3] Тригуб Р. М. Приближение функций с диофантовыми условиями многочленами с целыми коэффициентами // Метрические вопросы теории функций и отображений. Вып. 2. Киев: Наукова думка, 1971. С. 267-333.

[4] Ferguson B. O. Approximation by Polynomials with Integral Coefficients. Math. Surveys. V. 17. Providence (R.I.): Amer. Math. Soc., 1980.

[5] Тригуб Р. М. Прямые теоремы о приближении алгебраическими полиномами гладких функций на отрезке // Матем. заметки. 1993. Т. 54. №6. С. 113-121.

[6] Toland J.F. Self-adjoint operators and cones // J. London Math. Soc. 1996. V. 2. № 53. P. $167-183$.

[7] Trigub R. M. On the approximation of functions by polynomials with positive coefficients // East J. Approximation. 1998. V. 4. № 3. P. 379-389.

[8] Теляковский С. А. Две теоремы о приближении функций алгебраическими многочленами // Матем. сб. 1966. Т. 70. № 2. С. 252-265.

[9] Гопенгауз И. Е. К теореме А. Ф. Тимана о приближении функций многочленами на конечном отрезке // Матем. заметки. 1967. Т. 1. № 2. С. 163-172.

[10] Тиман А.Ф. Теория приближения функций действительного переменного. М.: Физматгиз, 1960.

[11] Brudnyi Yu. A. Timans type result on approximation by algebraic polynomials // Operator Theory Adv. Appl. 1997. V. 98. P. 92-101. 